American Journal of Infectious Diseases 4 (1): 85-91, 2008

ISSN 1553-6203

(C) 2008 Science Publications

\title{
C-Terminal Region of Dengue Virus Nonstructural Protein 1 Is Involved in Endothelial Cell Cross-Reactivity via Molecular Mimicry
}

\author{
${ }^{1}$ Shu-Wen Wan, ${ }^{2}$ Chiou-Feng Lin, ${ }^{3}$ Mei-Chun Chen, ${ }^{3}$ Huan-Yao Lei, ${ }^{3}$ Hsiao-Sheng Liu, ${ }^{4}$ Trai-Ming Yeh, \\ ${ }^{5}$ Ching-Chuan Liu and ${ }^{3}$ Yee-Shin Lin \\ ${ }^{1}$ Institute of Basic Medical Sciences, ${ }^{2}$ Institute of Clinical Medicine, \\ ${ }^{3}$ Department of Microbiology and Immunology, ${ }^{4}$ Department of Medical Technology, \\ ${ }^{5}$ Department of Pediatrics, National Cheng Kung University Medical College, Tainan, Taiwan
}

\begin{abstract}
Infection with dengue virus (DV) causes diseases ranging from self-limited dengue fever to life-threatening dengue hemorrhagic fever and dengue shock syndrome. Vascular leakage, thrombocytopenia and bleeding are the clinical manifestations associated with dengue hemorrhage. We previously showed that anti-DV nonstructural protein 1 (NS1) antibodies (Abs) cross-reacted with endothelial cells. The potential target proteins on endothelial cell surface recognized by anti-DV NS1 Abs showed sequence homology with the C-terminal amino acids (a.a.) 311-352 of DV NS1. In this study, the role of NS1 C-terminal region in dengue autoimmunity was investigated. We deleted the a.a. 277-352 of DV NS1 to prepare truncated NS1 (tNS1) and generated anti-DV tNS1 Abs in mice. The endothelial cell-binding activity of anti-DV tNS1 Abs was lower than that of anti-DV NS1 Abs. In addition, the endothelial cell-binding activity of anti-DV NS1 Abs was inhibited by preabsorption with DV NS1 but not with DV tNS1 proteins. The anti-P311 (a.a. 311-330) and anti-P331 (a.a. 331-350) titers of dengue patient sera were positively correlated with their endothelial cell-binding activity. Dengue patient sera showed lower binding activity to DV tNS1 than to DV NS1 proteins. The endothelial cell-binding activity of dengue patient sera was inhibited by preabsorption with P311 and P331. This study helps to understand the molecular mechanisms of autoimmunity mediated by anti-DV NS1 Abs and to provide the potential implications of tNS1 in dengue vaccine strategies.
\end{abstract}

Key words: Autoantibody, dengue virus, endothelial cells, molecular mimicry, NS1

\section{INTRODUCTION}

Dengue virus (DV) belongs to the family Flaviviridae and causes mild dengue fever (DF) or severe life-threatening diseases, including dengue hemorrhagic fever (DHF) and dengue shock syndrome $(\mathrm{DSS})^{[1-3]}$. Antibody-dependent enhancement (ADE) of infection poses a high risk for hemorrhage and shock syndrome, particularly in secondary heterotypic DV infection ${ }^{[4-7]}$. Aberrant immune responses to DV infection, such as the production of cytokines or chemokines, the activation of complement and immune cells and apoptotic cell death may also underlie the pathogenesis of dengue diseases ${ }^{[8-14]}$.

To date, no vaccine is available to prevent dengue disease. In order to avoid ADE, nonstructural protein 1 (NS1) has been proposed as a candidate for vaccine development. Both passive transfer of anti-DV NS1 antibodies (Abs) and active immunization of purified
NS1 or recombinant vaccinia virus expressing DV NS1 conferred protection in mice ${ }^{[15-17]}$. Protection by Abs to DV NS1 is thought to involve immune recognition of NS1 at the infected cell surface, followed by complement-mediated lysis ${ }^{[16]}$. DV NS1 is expressed in a glycosyl-phosphatidylinositol-linked form with a capacity for signal transduction, as evidenced by tyrosine phosphorylation of cellular proteins ${ }^{[18]}$. However, the cross-reactivity of anti-DV NS1 Abs with platelets and endothelial cells might cause thrombocytopenia and endothelial cell destruction ${ }^{[19-21]}$. These findings indicate the potential adverse effects of anti-DV NS1 Abs as a vaccine candidate. For the development of a safe vaccine, the DV NS1 epitopes that generate cross-reactive Abs will likely need to be deleted or mutated.

Using proteomic analysis and protein database screening, the C-terminal region of DV NS1 amino acid (a.a.) residues 311-352 showed sequence

Corresponding Author: Dr. Yee-Shin Lin, Department of Microbiology and Immunology, National Cheng Kung University Medical College, Tainan, Taiwan 
homology with several host proteins (Cheng et al., manuscript submitted). In this study, we determined whether the C-terminus of DV NS1 might be responsible for the molecular mimicry mechanism that results in the production of anti-endothelial autoAbs.

\section{MATERIALS AND METHODS}

Mice: $\mathrm{C} 3 \mathrm{H} / \mathrm{HeN}$ breeder mice were obtained from Charles River Breeding Laboratories and maintained on standard laboratory food and water in our medical college laboratory animal center. Their 8-week-old progeny were used for the experiments.

Patient sera: Serum samples were collected from patients with DF or DHF grades I-III during an outbreak in southern Taiwan in 2002. Diagnosis of DHF was based on the clinical criteria established by the World Health Organization.

Cell culture: Human microvascular endothelial cell line (HMEC-1 $)^{[22]}$ was passaged in culture plates containing endothelial cell growth medium M200 (Cascade Biologics) composed of 2\% fetal bovine serum, $1 \mu \mathrm{g} \mathrm{mL} L^{-1}$ hydrocortisone, $10 \mathrm{ng} \mathrm{mL}^{-1}$ epidermal growth factor, $3 \mathrm{ng} \mathrm{mL}{ }^{-1}$ basic fibroblast growth factor, $10 \mu \mathrm{g} \mathrm{mL} L^{-1}$ heparin and antibiotics. Cells were detached using $1000 \mathrm{U} \mathrm{mL}^{-1}$ trypsin and $0.5 \mathrm{mM}$ EDTA.

\section{Preparation of recombinant NS1 and truncated} NS1: The construction and production of recombinant full-length DV2 (New Guinea C strain) NS1 was performed as previously described ${ }^{[23]}$. C-terminus (a.a. residues 277-352)-deleted DV2 NS1 (truncated NS1; tNS1) gene was amplified by polymerase chain reaction (PCR) using pRSET-DV NS1 plasmid as the template. The forward primer (5'-CGGGATCCCGATAGTGGTTG-3') containing a Bam HI site and the reverse primer (5'-TCCCTCGAGGAAATCTCAGTCCATCTC-3') containing an $X h o$ I site were used. The cycles of PCR amplification was as follows: $95^{\circ} \mathrm{C}$ for $5 \mathrm{~min}, 30$ cycles of $95^{\circ} \mathrm{C}$ for $1 \mathrm{~min}, 60^{\circ} \mathrm{C}$ for $1 \mathrm{~min}, 72^{\circ} \mathrm{C}$ for 1 min and $72^{\circ} \mathrm{C}$ for $10 \mathrm{~min}$. The DNA fragments obtained were digested with Bam HI and Xho I and inserted into the vector pRSET (Invitrogen). After the sequence was confirmed, this plasmid was introduced into Escherichia coli BL21(DE3) pLys strain (Invitrogen). Remaining procedures were as previously described ${ }^{[23]}$.
Antibody preparation: Mice were immunized intraperitoneally with $25 \mu \mathrm{g}$ of recombinant full-length DV NS1 or DV tNS1 emulsified in complete Freund's adjuvant (Sigma) for one week and then four times in incomplete Freund's adjuvant. Sera were obtained 4 days after the last immunization. The IgG fractions from hyperimmunized mouse sera were purified with protein G-sepharose affinity chromatography column (Amersham).

ELISA: ELISA plates were coated with $100 \mu \mathrm{L}$ per well of $2 \mu \mathrm{g} \mathrm{mL}^{-1} \mathrm{NS} 1$ or tNS1 or $10 \mu \mathrm{g} \mathrm{mL}^{-1}$ peptides in coating buffer containing $0.015 \mathrm{M}$ sodium bicarbonate ( $\mathrm{pH} 9.6)$ and incubated overnight at $4^{\circ} \mathrm{C}$. Then, the plates were blocked overnight at $4^{\circ} \mathrm{C}$ with $200 \mu \mathrm{L}$ per well of $5 \%$ bovine serum albumin in coating buffer. The plates were washed three times with phosphate-buffered saline containing $0.05 \%$ Tween-20 (PBS-T). After three washes with PBS-T, $100 \mu \mathrm{L}$ per well of $1: 1000$ diluted patient sera or $2 \mu \mathrm{g}$ $\mathrm{mL}^{-1}$ anti-full-length DV NS1 or anti-DV tNS1 Abs were added and incubated overnight at $4^{\circ} \mathrm{C}$. After the plates were washed three times in PBS-T, $100 \mu \mathrm{L}$ per well of peroxidase-conjugated anti-human IgM or IgG or anti-mouse IgG was added and incubated for $2 \mathrm{~h}$ at room temperature. After three further washes, $100 \mu \mathrm{L}$ per well of 2, 2'azinobis 3-ethylbenzthiaoline sulfonic acid (Sigma) was added and analyzed using a microplate reader (Molecular Devices). The absorbance was measured at $405 \mathrm{~nm}$.

Flow cytometry: Cells were fixed with $1 \%$ formaldehyde in PBS at room temperature for $10 \mathrm{~min}$. After three washes with PBS, cells were incubated with 1:25 dilution of dengue patient sera or 2 or $5 \mu \mathrm{g}$ mouse anti-DV NS1 and anti-DV tNS1 Abs at $4^{\circ} \mathrm{C}$ for $1 \mathrm{~h}$. Following a further three washes with PBS, cells were incubated with $1 \mu \mathrm{L}$ of $1 \mathrm{mg} \mathrm{mL}^{-1}$ of FITC-conjugated goat anti-human $\operatorname{IgG}$ or $\operatorname{IgM}$ or donkey anti-mouse $\mathrm{IgG}$ at $4^{\circ} \mathrm{C}$ for $1 \mathrm{~h}$ and analyzed by flow cytometry (FACSCalibur; BD Biosciences) with excitation set at $488 \mathrm{~nm}$.

Statistics: We used the paired $t$-test for statistical analysis. Statistical significance was set at $\mathrm{p}<0.05$.

\section{RESULTS}

We previously showed that anti-DV NS1 Abs cross-reacted with endothelial cells ${ }^{[21,23]}$. The potential target proteins recognized by anti-DV NS1 Abs were 
identified by proteomic analysis (Cheng et al., manuscript submitted). The sequence alignment showed homology of C-terminal region a.a. 311-352 of DV NS1 with the candidate proteins.

To study the role of the C-terminus of DV NS1 in the cross-reactivity of anti-DV NS1 Abs with endothelial cells, we deleted the a.a. 277-352 of DV NS1 to prepare truncated NS1 (tNS1). As shown in Fig. 1A, recombinant DV NS1 and tNS1 proteins have estimated molecular masses of 46 and $35 \mathrm{kDa}$ for DV NS1 and tNS1, respectively. The binding activities of anti-DV NS1 and anti-DV tNS1 Abs from hyperimmunized mouse sera with recombinant proteins (DV NS1 and tNS1) and peptides (P311: a.a. 311-330 and P331: a.a. 331-350) were determined by ELISA. Anti-DV NS1 IgG showed less binding to DV tNS1 than to NS1, whereas the binding activities of anti-DV tNS1 IgG to DV NS1 and tNS1 were similar (Fig. 1B). In addition, anti-DV tNS1 IgG showed only basal levels of binding with P311 and P331 (Fig. 1C).

We then investigated the binding activity of anti-DV NS1 and anti-DV tNS1 Abs with endothelial cells. The cross-reactivity of anti-tNS1 Abs with HMEC-1 cells was lower than that of anti-NS1 Abs as viewed by immunostaining and confocal microscopy (Fig. 2A). The quantitative results as analyzed by flow cytometry confirmed a lower HMEC-1 binding activity of anti-tNS1 Abs as compared with that of anti-NS1 Abs (Fig. 2B). By preabsorption assay, NS1 protein, but not tNS1, inhibited the binding activity of anti-DV NS1 to HMEC-1 cells (Fig. 2C).

We next assayed the binding activity of dengue patient sera with DV NS1 and tNS1 proteins. Antibody binding using individual DF (Fig. 3A) and DHF (Fig. 3B) patient sera were determined. We found that the tNS1 binding activity of patient sera IgM was lower than the NS1 binding activity in DF and DHF patient sera as summarized in Fig. 3C.

We subsequently determined whether the cross-reactive Abs in dengue patient sera were directed against P311 or P331. The HMEC-1 cell binding activity of dengue patient sera $\operatorname{IgM}$ was positively correlated with the anti-NS1 IgM titers in both DF and DHF patients. The correlation of HMEC-1 cell binding activity with anti-P311 and anti-P331 titers was higher in DHF patient sera than in DF patient sera (Fig. 4A). There was no correlation of anti-P311 and anti-P331 IgG titers with the HMEC-1 cell binding activity of dengue patient sera IgG (data

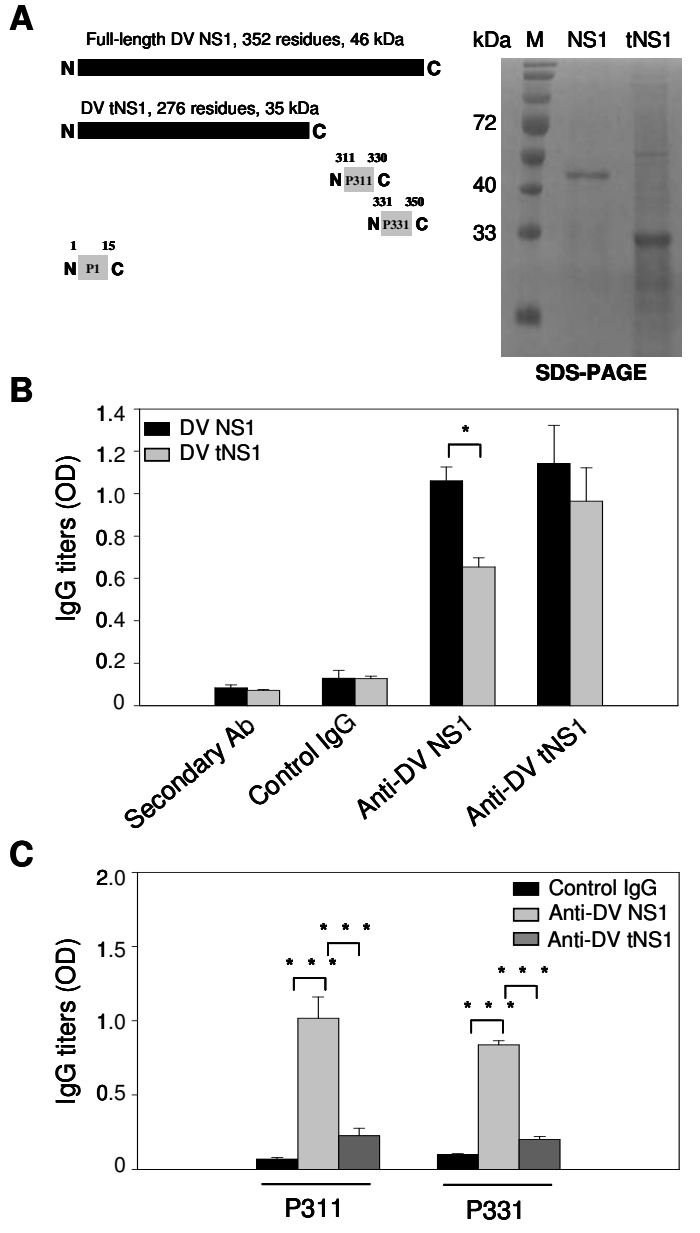

Fig. 1: The generation of DV tNS1 and the binding activity of anti-DV tNS1 Abs. (A) The diagram shows a linear depiction of the recombinant DV NS1 and tNS1 proteins and peptides (P311: a.a. 311-330, P331: a.a. 331-350, and P1: a.a. 1-15). Recombinant DV NS1 and tNS1 proteins from E. coli expression system are confirmed by SDS-PAGE. M: molecular weight marker. (B) The binding activity of anti-DV NS1 Abs with DV tNS1 was lower than that with NS1, whereas the binding activity of anti-DV tNS1 Abs with DV NS1 and tNS1 had similar levels. (C) The reactivity of anti-DV NS1 and anti-DV tNS1 Abs with P311 and P331. The O.D.405nm values are shown. *: $\mathrm{p}<0.05, * * *$ : $\mathrm{p}<0.001$ 
A

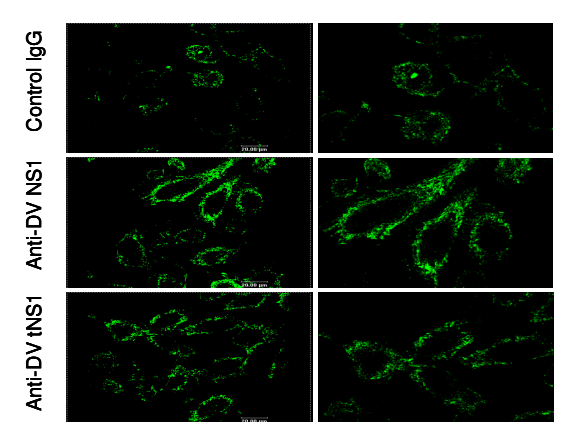

B

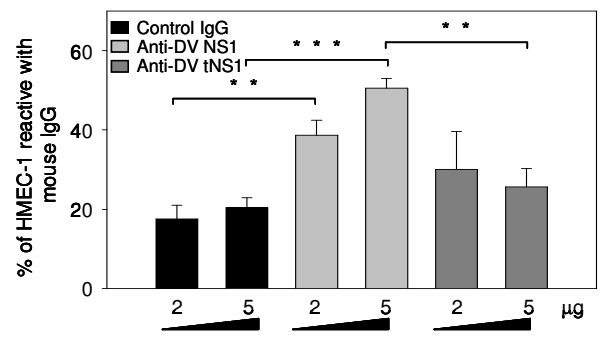

C

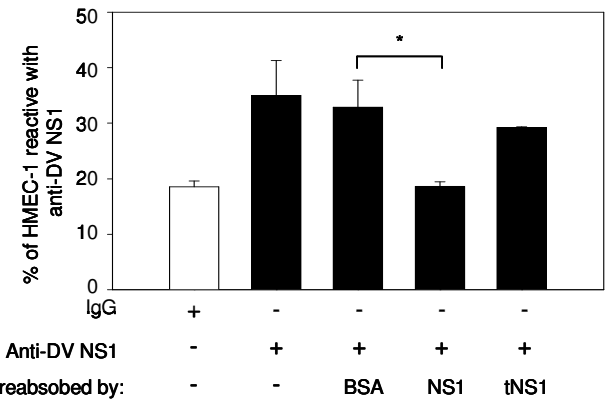

Fig. 2: The role of the C-terminus of DV NS1 for the cross-reactivity of anti-NS1 Abs with endothelial cells. The binding activities of anti-DV NS1 and anti-DV tNS1 Abs were detected by fluorescence microscopy (A) and flow cytometry (B). The HMEC-1 cell binding activity of anti-DV tNS1 Abs was lower than that of anti-DV NS1 Abs. (C) The HMEC-1 cell binding activity of anti-DV NS1 Abs was inhibited by preabsorption with DV NS1 but not with tNS1 proteins. Preabsorption with bovine serum albumin (BSA) was used as a negative control. *: $\mathrm{p}<0.05, * *: \mathrm{p}<0.01, * * *: \mathrm{p}<0.001$
A

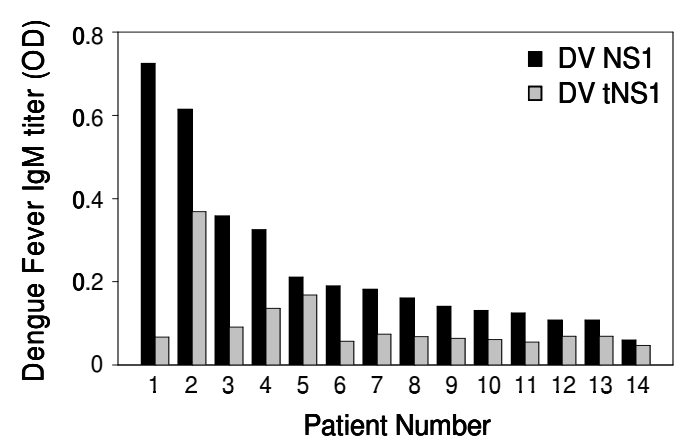

B

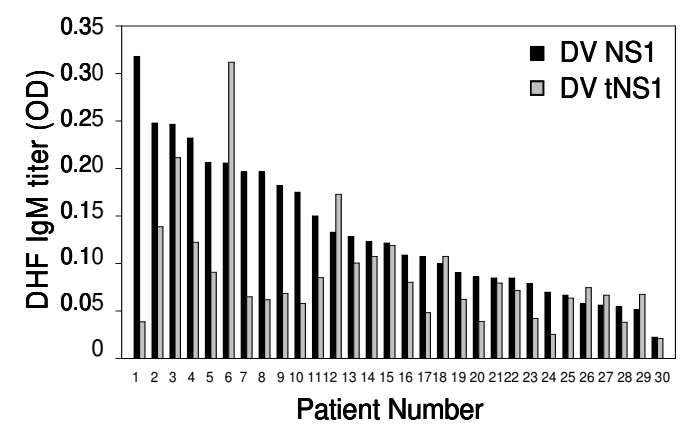

C

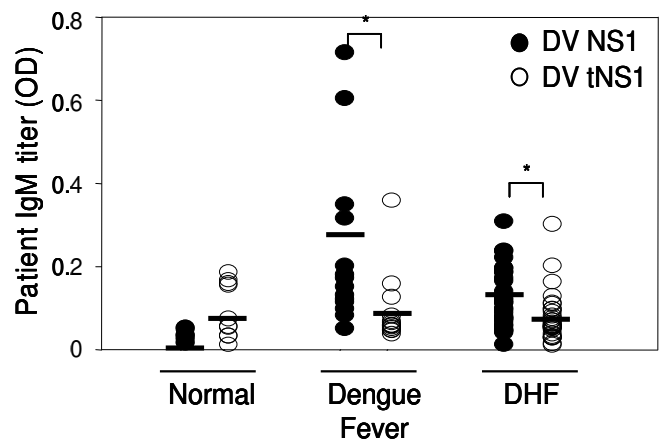

Fig. 3: Dengue patient sera show lower binding activity to DV tNS1 than with DV NS1 proteins. The binding activities of DF (A) and DHF (B) individual patient sera IgM with DV NS1 and tNS1 were determined by ELISA. The average binding activities of normal ( $\mathrm{n}=$ 10), DF $(\mathrm{n}=14)$, and DHF $(\mathrm{n}=30)$ patient sera with DV NS1 and tNS1 proteins are shown in (C). *: p<0.05 
A

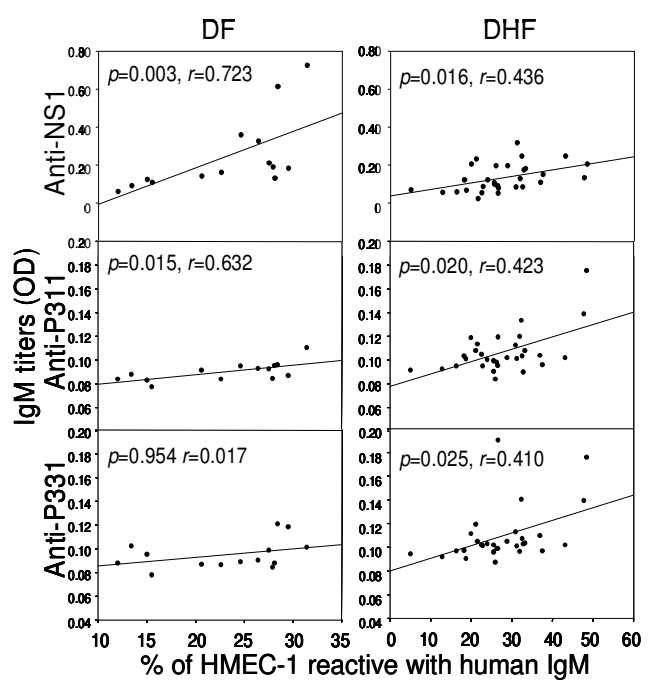

B

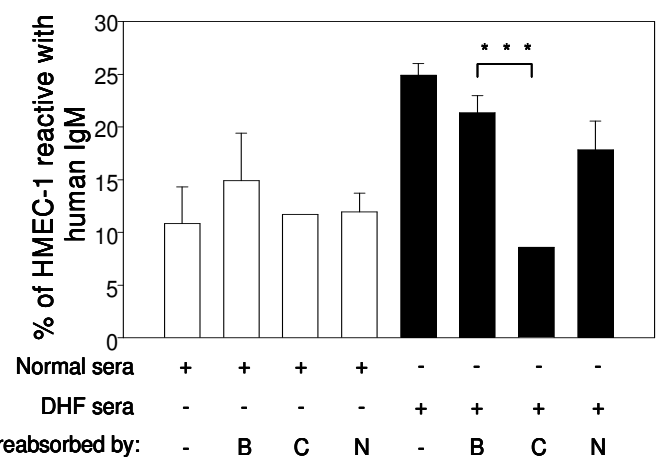

Fig. 4: The role of the C-terminus of DV NS1 in the cross-reactivity of dengue patient sera with endothelial cells. (A) The titers of anti-DV NS1, anti-P311, and anti-P331 IgM in dengue patient sera were detected by ELISA. The HMEC-1 cell binding activity of patient sera were analyzed by flow cytometry. The correlation of the binding activity to HMEC-1 cells and the anti-DV NS1, anti-P311 or anti-P331 IgM titers in DF (left panels) and DHF (right panels) patient sera are shown. (B) DHF patient sera (from a pool of three patients) or normal sera (from a pool of three healthy controls) were preabsorbed with BSA, the C-terminal peptide mixture of P311 and P331, or the N-terminal peptide P1. Their binding activity with HMEC-1 cells was detected by flow cytometry. $* * *: p<0.001$ not shown). To further confirm the role of the NS1 C-terminus, HMEC-1 cells were incubated with DHF patient sera (from a pool of three patients) or normal sera (from a pool of two healthy controls) which were preabsorbed with BSA, C-terminal P311 and P331 peptide mixture, or N-terminal P1 (a.a. 1-15). The endothelial cell-binding activity of dengue patient sera was inhibited by preabsorption with a mixture of P311 and P331, but not with BSA or P1 (Fig. 4B). Therefore, the C-terminal a.a. 311-352 of DV NS1 play an important role in the cross-reactivity of anti-NS1 Abs with endothelial cells in DV infection.

\section{DISCUSSION}

DV infection is an important human disease due to its widespread distribution with incumbent morbidity and mortality. It is also a disease involving complex pathogenic mechanisms, including ADE and other immunopathological responses ${ }^{[24,25]}$. We previously showed a mechanism of molecular mimicry in which Abs against DV NS1 cross-reacted with endothelial cells and caused damage ${ }^{[21,23]}$. In the present study, we further demonstrated that the C-terminal region of DV NS1 plays an important role in endothelial cell cross-reactivity.

Autoimmunity characterized by the generation of autoAbs and the activation of autoreactive lymphocytes has been demonstrated in a number of viral infections, such as human immunodeficiency virus, hepatitis $\mathrm{C}$ virus, cytomegalovirus, herpes simplex virus and Epstein-Barr virus ${ }^{[26-32]}$. The generation of autoAbs cross-reactive with endothelial cells is involved in several autoimmune vascular diseases $^{[33-35]}$. Our studies ${ }^{[23,36-38]}$ showed that Abs directed against DV NS1 cross-reacted with endothelial cells and induced these cells to undergo nitric oxide-mediated apoptosis as well as cytokine and chemokine production. The onset of autoimmune responses leading to endothelial barrier disruption may have implications for the pathogenesis of dengue hemorrhagic syndrome. In the present study, we found a positive correlation between the anti-NS1 titers in dengue patient sera and their endothelial cell-binding activity. However, the positive correlation pattern was only observed with antibodies of the IgM, but not IgG, isotype. In other words, although dengue patients might produce high titers of anti-NS1 Abs, the endothelial cell cross-reactive anti-NS1 are predominantly IgM. This is consistent with our previous finding that the percentages of endothelial cells reactive with patient sera IgM were higher than those with $\operatorname{IgG}^{[21]}$. Nevertheless, the involvement of anti-NS1 IgG in endothelial cell binding can not be excluded. In addition, our studies using purified IgG from NS1-hyperimmunized mouse sera showed endothelial cell cross-reactivity. 
In order to confirm a mechanism of molecular mimicry, we have identified several endothelial cell surface molecules recognized by anti-DV NS1 Abs. Furthermore, we predicted the presence of cross-reactive epitope(s) in the $\mathrm{C}$-terminal region of NS1 (Cheng et al., manuscript submitted). There were good correlations of endothelial cell-binding activity with anti-P311 (a.a. 311-330) and anti-P331 (a.a. 331-350) titers in DHF patient sera. We deleted the C-terminal end of a.a. 277-352 and found that anti-tNS1 had reduced binding activity to endothelial cells. In addition, dengue patient sera showed lower binding activity to DV tNS1 than to DV NS1 proteins. The NS1 C-terminal deletion at a.a. residue 277 was predicted not to cause conformational changes based on the disulfide bond arrangement ${ }^{[39]}$. However, this prediction needs to be further confirmed.

Both anti-E and anti-prM Abs may enhance DV infection in FcR-bearing cells or in cells without $\mathrm{FcR}^{[24,40]}$. In order to avoid the ADE effect, NS1 has been considered as a candidate for vaccine development. However, a caveat may lie in the observations that anti-DV NS1 Abs bind to platelets and endothelial cells and cause pathogenic effects ${ }^{[19-21]}$ Here, we show that tNS1 may hold promise as a subunit vaccine candidate. Studies are in progress to further confirm that tNS1 does not cause pathologic effects in vitro and in vivo. Also, the protective effects conferred by tNS1 protein or DNA vaccine remain to be evaluated. The protection of NS1-DNA vaccine can be augmented, for example, by co-injection of plasmid encoding interleukin-12 as previously suggested ${ }^{[41]}$. This approach awaits further investigation.

\section{ACKNOWLEDGMENT}

This work was supported by grant NSC95-3112-B006-002 from the National Research Program of Genomic Medicine, National Science Council, Taiwan. We thank Dr. Robert Anderson for critical reading of this manuscript.

\section{REFERENCES}

1. Henchal, E.A. and J.R. Putnak, 1990. The dengue viruses. Clin. Microbiol. Rev., 3: 376-396.

2. Monath, T.P., 1994. Dengue: The risk to developed and developing countries. Proc. Natl. Acad. Sci. USA, 91: 2395-2400.

3. Gubler, D.J., 1998. Dengue and dengue hemorrhagic fever. Clin. Microbiol. Rev., 11: 480-496.

4. Halstead, S.B., 1988. Pathogenesis of dengue: challenges to molecular biology. Science, 239: 476-481.

5. Halstead, S.B., 2002. Dengue. Curr. Opin. Infect. Dis., 15: 471-476.
6. Mady, B.J., D.V. Erbe, I. Kurane, M.W. Fanger and F.A. Ennis, 1991. Antibody-dependent enhancement of dengue virus infection mediated by bispecific antibodies against cell surface molecules other than Fc $\gamma$ receptors. J. Immunol., 147: 3139-3144.

7. Morens, D.M., 1994. Antibody-dependent enhancement of infection and the pathogenesis of viral disease. Clin. Infect. Dis., 19: 500-512.

8. Rothman, A.L. and F.A. Ennis, 1999. Immunopathogenesis of dengue hemorrhagic fever. Virology, 257: 1-6.

9. Lei, H.Y., T.M. Yeh, H.S. Liu, Y.S. Lin, S.H. Chen and C.C. Liu, 2001. Immunopathogenesis of dengue virus infection. J. Biomed. Sci., 8: 377-388.

10. Malavige G.N., S. Fernando, D.J. Fernando and S.L. Seneviratne, 2004. Dengue viral infections. Postgrad. Med. J., 80: 588-601.

11. Green, S. and A. Rothman, 2006. Immunopathological mechanisms in dengue and dengue hemorrhagic fever. Curr. Opin. Infect. Dis., 19: 429-436.

12. Clyde K, J.L. Kyle and E. Harris, 2006. Recent advances in deciphering viral and host determinants of dengue virus replication and pathogenesis. J. Virol., 80: 11418-11431.

13. Avirutnan, P., P. Malasit, B. Seliger, S. Bhakdi and M. Husmann, 1998. Dengue virus infection of human endothelial cells leads to chemokine production, complement activation and apoptosis. J. Immunol., 161: 6338-6346.

14. Mongkolsapaya, J., W. Dejnirattisai, X.N. Xu, S. Vasanawathana, N. Tangthawornchaikul, A. Chairunsri, S. Sawasdivorn, T. Duangchinda, T. Dong, S. Rowland-Jones, P. Yenchitsomanus, A. McMichael, P. Malasit and G. Screaton, 2003. Original antigenic sin and apoptosis in the pathogenesis of dengue hemorrhagic fever. Nat. Med., 9: 921-927.

15. Henchal, E.A., L.S. Henchal and J.J. Schlesinger, 1988. Synergistic interactions of anti-NS1 monoclonal antibodies protect passively immunized mice from lethal challenge with dengue 2 virus. J. Gen. Virol., 69: 2101-2107.

16. Schlesinger, J.J., M.W. Brandriss and E.E. Walsh, 1987. Protection of mice against dengue 2 virus encephalitis by immunization with the dengue 2 virus non-structural glycoprotein NS1. J. Gen. Virol., 68: 853-857.

17. Falgout, B., M. Bray, J.J. Schlesinger and C.J. Lai, 1990. Immunization of mice with recombinant vaccinia virus expressing authentic dengue virus nonstructural protein NS1 protects against lethal dengue virus encephalitis. J. Virol., 64: 4356-4363. 
18. Jacobs, M.G., P.J. Robinson, C. Bletchly, J.M. Mackenzie and P.R. Young, 2000. Dengue virus nonstructural protein 1 is expressed in a glycosyl-phosphatidylinositol-linked form that is capable of signal transduction. FASEB J., 14: 1603-1610.

19. Falconar, A.K.I., 1997. The dengue virus nonstructural-1 protein (NS1) generates antibodies to common epitopes on human blood clotting, integrin adhesin ${ }^{-1}$ proteins and binds to human endothelial cells: potential implications in haemorrhagic fever pathogenesis. Arch. Virol., 142: 897-916.

20. Lin, C.F., H.Y. Lei, C.C. Liu, H.S. Liu, T.M. Yeh, S.T. Wang, T.I. Yang, F.C. Sheu, C.F. Kuo and Y.S. Lin, 2001. Generation of IgM anti-platelet autoantibody in dengue patients. J. Med. Virol., 63: 143-149.

21. Lin, C.F., H.Y. Lei, A.L. Shiau, C.C. Liu, H.S. Liu, T.M. Yeh, S.H. Chen and Y.S. Lin, 2003. Antibodies from dengue patient sera cross-react with endothelial cells and induce damage. J. Med. Virol., 69: 82-90.

22. Ades, E.W., F.J. Candal, R.A. Swerlick, V.G. George, S. Summers, D.C. Bosse and T.J. Lawley, 1992. HMEC-1: establishment of an immortalized human microvascular endothelial cell line. J. Invest. Dermatol., 99: 683-690.

23. Lin, C.F., H.Y. Lei, A.L. Shiau, H.S. Liu, T.M. Yeh, S.H. Chen, C.C. Liu, S.C. Chiu and Y.S. Lin, 2002. Endothelial cell apoptosis induced by antibodies against dengue virus nonstructural protein 1 via production of nitric oxide. $\mathrm{J}$. Immunol., 169: 657-664.

24. Halstead, S.B., 2003. Neutralization and antibody-dependent enhancement of dengue viruses. Adv. Virus Res., 60: 421-467.

25. Rothman, A.L., 2004. Dengue: defining protective versus pathologic immunity. J. Clin. Invest., 113: 946-951.

26. Kaplan, C., F. Morinet and J. Cartron, 1992. Virus-induced autoimmune thrombocytopenia and neutropenia. Semin. Hematol., 29: 34-44.

27. Asherson, R.A. and R. Cervera, 2003. Antiphospholipid antibodies and infections. Ann. Rheum. Dis., 62: 388-393.

28. Zandman-Goddard, G. and Y. Shoenfeld, 2002. HIV and autoimmunity. Autoimmun. Rev., 1: 329-337.

29. Rouse, B.T. and S. Deshpande, 2002. Viruses and autoimmunity: an affair but not a marriage contract. Rev. Med. Virol., 12: 107-113.

30. Molina, V. and Y. Shoenfeld, 2005. Infection, vaccines and other environmental triggers of autoimmunity. Autoimmunity, 38: 235-245.
31. Fujinami, R.S., M.G. von Herrath, U. Christen and J.L. Whitton, 2006. Molecular mimicry, bystander activation, or viral persistence: infections and autoimmune disease. Clin. Microbiol. Rev., 19: 80-94.

32. Kim, B., S.D. Kaistha and B.T. Rouse, 2006. Viruses and autoimmunity. Autoimmunity, 39: 71-77.

33. Renaudineau, Y., C. Dugue, M. Dueymes and P. Youinou, 2002. Antiendothelial cell antibodies in systemic lupus erythematosus. Autoimmun. Rev., 1: 365-372.

34. Armitage, J.D., S. Homer-Vanniasinkam and N.J. Lindsey, 2004. The role of endothelial cell reactive antibodies in peripheral vascular disease. Autoimmun. Rev., 3: 39-44.

35. Meroni, P., N. Ronda, E. Raschi and M.O. Borghi, 2005. Humoral autoimmunity against endothelium: theory or reality? Trends Immunol., 26: 275-281.

36. Lin, C.F., S.C. Chiu, Y.L. Hsiao, S.W. Wan, H.Y. Lei, A.L. Shiau, H.S. Liu, T.M. Yeh, S.H. Chen, C.C. Liu and Y.S. Lin, 2005. Expression of cytokine, chemokine and adhesion molecules during endothelial cell activation induced by antibodies against dengue virus nonstructural protein 1. J. Immunol., 174: 395-403.

37. Lin, Y.S., C.F. Lin, H.Y. Lei, H.S. Liu, T.M. Yeh, S.H. Chen and C.C. Liu, 2004. Antibody-mediated endothelial cell damage via nitric oxide. Curr. Pharm. Design, 10: 213-221.

38. Lin, C.F., S.W. Wan, H.J. Cheng, H.Y. Lei and Y.S. Lin, 2006. Autoimmune pathogenesis in dengue virus infection. Viral Immunol., 19: 127-132.

39. Wallis, T.P., C.Y. Huang, S.B. Nimkar, P.R. Young and J.J. Gorman, 2004. Determination of the disulfide bond arrangement of dengue virus NS1 protein. J. Biol. Chem., 279: 20729-20741.

40. Huang, K.J., Y.C. Yang, Y.S. Lin, J.H. Huang, H.S. Liu, T.M. Yeh, S.H. Chen, C.C. Liu and H.Y. Lei, 2006. The dual-specific binding of dengue virus and target cells for the antibody-dependent enhancement of dengue virus infection. J. Immunol., 176: 2825-2832.

41. Wu, S.F., C.L. Liao, Y.L. Lin, C.T. Yeh, L.K. Chen, Y.F. Huang, H.Y. Chou, J.L. Huang, M.F. Shaio and H.K. Sytwu, 2003. Evaluation of protective efficacy and immune mechanisms of using a non-structural protein NS1 in DNA vaccine against dengue 2 virus in mice. Vaccine, 21: 3919-3929. 